\title{
Patient's experiences of involuntary hospital admission and treatment: A review of qualitative studies
}

\author{
CHRISTINA KATSAKOU and STEFAN PRIEBE \\ Unit for Social \& Community Psychiatry, Barts and the London School of Medicine, Queen Mary, University of London, \\ London (United Kingdom)
}

\begin{abstract}
SUMMARY. Aims - This study aimed to explore psychiatric patients' experiences of involuntary admission and treatment by reviewing qualitative studies. Method - Qualitative studies investigating patients' experiences of involuntary treatment were identified. Relevant databases were searched and authors were contacted. Thematic analysis was applied for the synthesis of emerging issues. Results - Five studies fulfilled the inclusion criteria. The main areas that appear to be of importance are: patients' perceived autonomy and participation in decisions for themselves, their feeling of whether or not they are being cared for and their sense of identity. In these areas both negative and positive consequences from involuntary admission were mentioned. However, methodological weaknesses were also found, such as small sample sizes. Furthermore, it is not described whether these themes are mentioned by all participants as negative and positive aspects of their experience or whether they reflect views supported by distinct groups. Conclusions - Although the perceived impact of involuntary treatment is fairly clearly described, differences between distinct patient groups are not examined. Future research should investigate such differences in order to inform relevant policy decisions for particular groups.
\end{abstract}

Declaration of Interest: None.

KEY WORDS: Commitment of the mentally ill, patient admission, coercion, qualitative research.

Received 20.12.2006 - Final version received 02.02.2007 - Accepted on 02.02.2007.

\section{INTRODUCTION}

Involuntary hospital admissions are practiced more or less throughout the entire world. Issues around the justification, legal regulation and best practice of compulsory interventions in mental health care have been widely questioned and discussed. Researchers have also shown interest in the area and several quantitative studies have been conducted in this field. Findings from a recent review of quantitative literature on involuntary admission (Katsakou \& Priebe, 2006) suggest that on average, involuntarily admitted patients show clinical improvement and retrospectively view their admission rather positively. However, a substantial proportion of patients continue to feel that their admission was neither justified nor beneficial. Although quantitative research findings offer a numerical representation of patients' views and preferences, qualitative research can offer a more detailed description of people's experiences, identifying positive and negative aspects and/ or patient groups with more or less favourable opinions. Therefore, a review of qualitative studies in the area of involuntary treatment was considered appropriate.

Hence, the present review explores qualitative evidence on involuntary admission in general adult psychiatry and focuses on the following questions:

1. How do patients experience involuntary admission and treatment and what are their perceived outcomes of such interventions?

2. Do patients report both positive and negative experiences and how are these linked?

3. What are the differences between patient groups holding negative and positive views on this issue in terms of their characteristics, thought processes and treatment experiences?
Address for correspondence: Professor S. Priebe, Academic Unit, Newham Centre for Mental Health, London E13 8SP (United Kingdom). Fax: 0 (+44) 2075402976 E-mail: s.priebe@qmul.ac.uk

\section{METHOD}

A literature search was conducted in July 2006 in the following electronic databases: Psychinfo, Medline, 
Premedline, Embase, using two types of keywords: the first referred to the topic under study, using the following keywords: compulsory/ involuntary/ coercion/ forced/ commitment/ detention/ admission/ treatment/ hospitalisation and the second covered a range of qualitative methodologies, i.e. qualitative, grounded theory, phenomenology, ethnography, thematic analysis, content analysis. The two types of keywords were exploded and combined in the databases. References within each article were also searched in order to identify more relevant papers. Personal correspondence with authors and other experts in the area, such as service user groups' representatives, helped to locate more relevant articles and/ or gain additional information in the already identified studies.

The following inclusion and exclusion criteria were used: Studies were included if they a) had assessed the experiences of involuntarily admitted patients, b) included patients who had been treated in acute general psychiatric wards, c) used a qualitative methodology, and d) were published in English.

Studies were excluded if they a) were conducted in units for eating disorders, forensic or drug addiction units, b) assessed mixed samples of involuntary and voluntary patients, with no separate analysis for the involuntary group, c) explored the views of patients treated involuntarily in the community rather than in a hospital setting d) only explored experiences of specific coercive measures (eg. Seclusion) and not overall views on involuntary admission and treatment.

Five quality checklists for reviewing qualitative research were reviewed and adapted in order to devise an extraction sheet to aid the systematic abstraction of information and evaluation of the qualitative investigations reviewed here (Dixon- Woods et al., 2004; Law et al., 1998; Bromley et al., 2002; Barbour, 2001; Milton Keynes Primary Care Trust, 2002: Clark, 2003; DixonWoods \& Fitzpatrick, 2001; Seale, 2002).

Thematic analysis was used to facilitate the synthesis of results from the reviewed studies. After reading all studies, a coding template was devised based on all themes and subthemes identified in each study. Using the constant comparative method, themes and subthemes were revised and organised under broader categories, which are presented in detail in the results section.

The main reviewer has a background in psychology and is therefore particularly interested in psychological processes, subjective perspectives and experiences, as well as underlying mechanisms that might further illuminate participants' views. In addition, her understandings and interpretations are mostly shaped by academic interests and patients' subjective meanings rather than by clinical experience or professionals' needs and perspectives. The second reviewer has a background on psychology and psychiatry, as well as wide experience in the areas of research and clinical practice.

\section{RESULTS}

The search yielded 188 papers. Once the titles of all articles were reviewed, only 33 papers were considered relevant. Abstracts for these papers were obtained and read, but only 5 papers met the inclusion criteria (Olofsson \& Jacobsson, 2001; Olofsson \& Norberg, 2001; Johansson \& Lundman, 2002; Jones \& Mason, 2002; Quirk et al., 2003). These articles reported findings from 4 studies. Further communication with one of the authors identified a report of the published study (Quirk et al., 2003) to the funding body (Department of Health) that contained more detailed information relevant to this review. Further contact with user researchers' groups in the U.K. identified a report to the funding body of a userled investigation in this area that had not been published (Barnes et al., 2000).

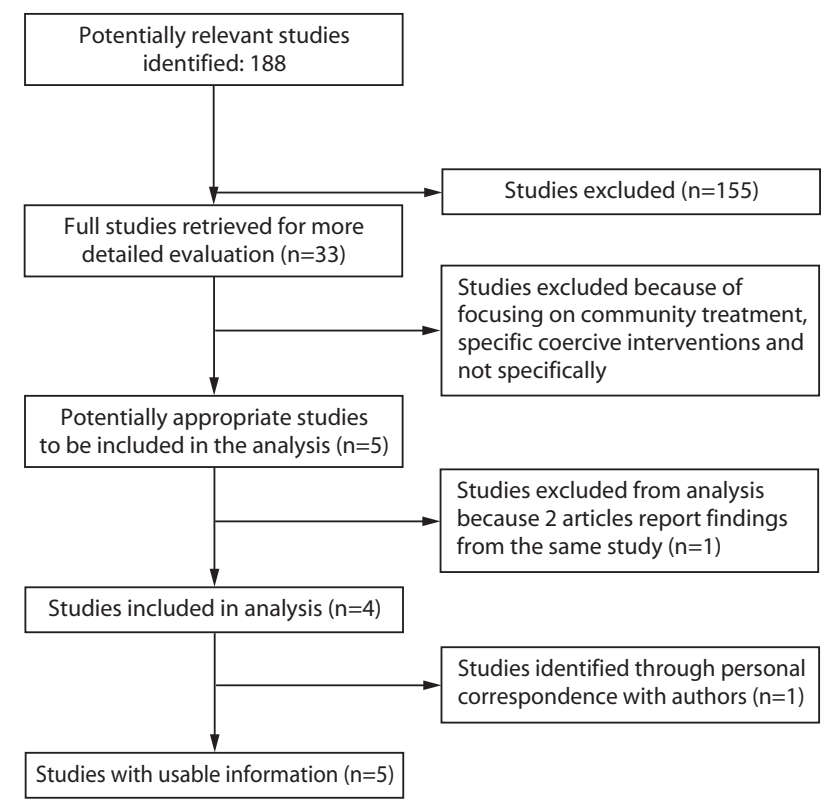

Figure 1. - Identifying relevant studies: adapted from the QUOROM statement (Clarke, 2000). 


\section{Study methodology}

All five studies used semi-structured interviews to assess patients' opinions on various aspects of their involuntary treatment. Various methodological approaches were followed to guide the methods and analysis in each study, including phenomenological interpretative analysis, narrative analysis, and grounded theory.

\section{Study aims- research questions}

Each study explored slightly different aspects of involuntary patients' experiences. The research aims and questions were not always clearly stated, but the main areas of interest in the 5 studies are described in Table I.

\section{Sample}

The sample sizes range from 4 to 18 participants and the response rates of those who agreed to participate after being approached by researchers are only reported in 2 studies (Quirk et al., 2000; Olofsson \& Jacobsson, 2001 ) and range from $28 \%$ to $58 \%$. The samples are either consecutive or convenient. The total number of participants in all included studies is 54. All participants had experienced involuntary admission to a psychiatric hospital but were recruited through different paths, such as hospital staff, community mental health teams and service users' community groups. They were interviewed in various settings, either in the hospital or in community services. Two studies were conducted in Sweden and 3 in the U.K.

\section{Analysis}

Most studies (4/5) claim that they have followed specific approaches in qualitative research for data analysis and interpretation. However, the specific analytical steps followed, the coding and the synthesis of evidence are not described in detail. Only limited quotes from the raw data are presented. In addition, no inter-rater reliability was performed for the coding of the interviews, although discussion of themes among more than one researchers is reported in all studies. All interviewers are independent and not involved in the interviewees' care in any way. Nevertheless, although the background of the researchers is mentioned in most studies (3/5), the way this could affect data interpretation is not discussed in any of the studies.

\section{Thematic analysis of results}

In this synthesis the main findings from all five studies describing patients' experiences under different stages of involuntary care and various forms of coercion were combined and organised under common themes. These themes are presented below. As involuntary care was initially experienced rather negatively than positively, the themes that refer to negative aspects of the whole experience are listed first and are then followed by positive aspects.

\section{Negative aspects:}

a) Restrictions of autonomy/no participation in decisions for treatment:

One of the most commonly mentioned issues refers to experiences of violations of autonomy. Participants feel that their rights are taken away; they are given no option and have no say in decisions regarding their treatment. "It was an imposition of treatment- an abuse of human rights" (Barnes et al., 2000). They receive no information on their treatment and their views are ignored. People report feeling that they are not allowed to be responsible for themselves. They are locked in a place where the care they receive is dominated by strict rules they do not understand. There is no flexibility and no room for participation in their own treatment. Other people, such as the police and health professionals, have power over them. Sometimes, they even experience physical violations and coercion, when they are restrained, secluded or given forced medication against their will. "...I become aggressive when they use violence, it's an encroachment when they don't say anything but just catch hold of you and drag to the bed and give the injection with force and a lot of people are holding you. They didn't have to use violence, they didn't need a whole army from two wards..." (Johansson \& Lundman, 2002). This situation can be frustrating and often leads to feelings of failure and powerlessness. There is a dominating theme of loss, as people feel they lose their liberty, their rights, the power to decide for themselves and cannot have control over their lives anymore.

\section{b) Quality of care/ not being cared for:}

People's overall views on their involuntary admission are linked to their experiences of care in the hospital. Patients often report that the treatment they receive is meaningless and not appropriate. "I think there is no point in being locked in as nothing is happening there, 
Patient's experiences of involuntary hospital admission and treatment: A review of qualitative studies

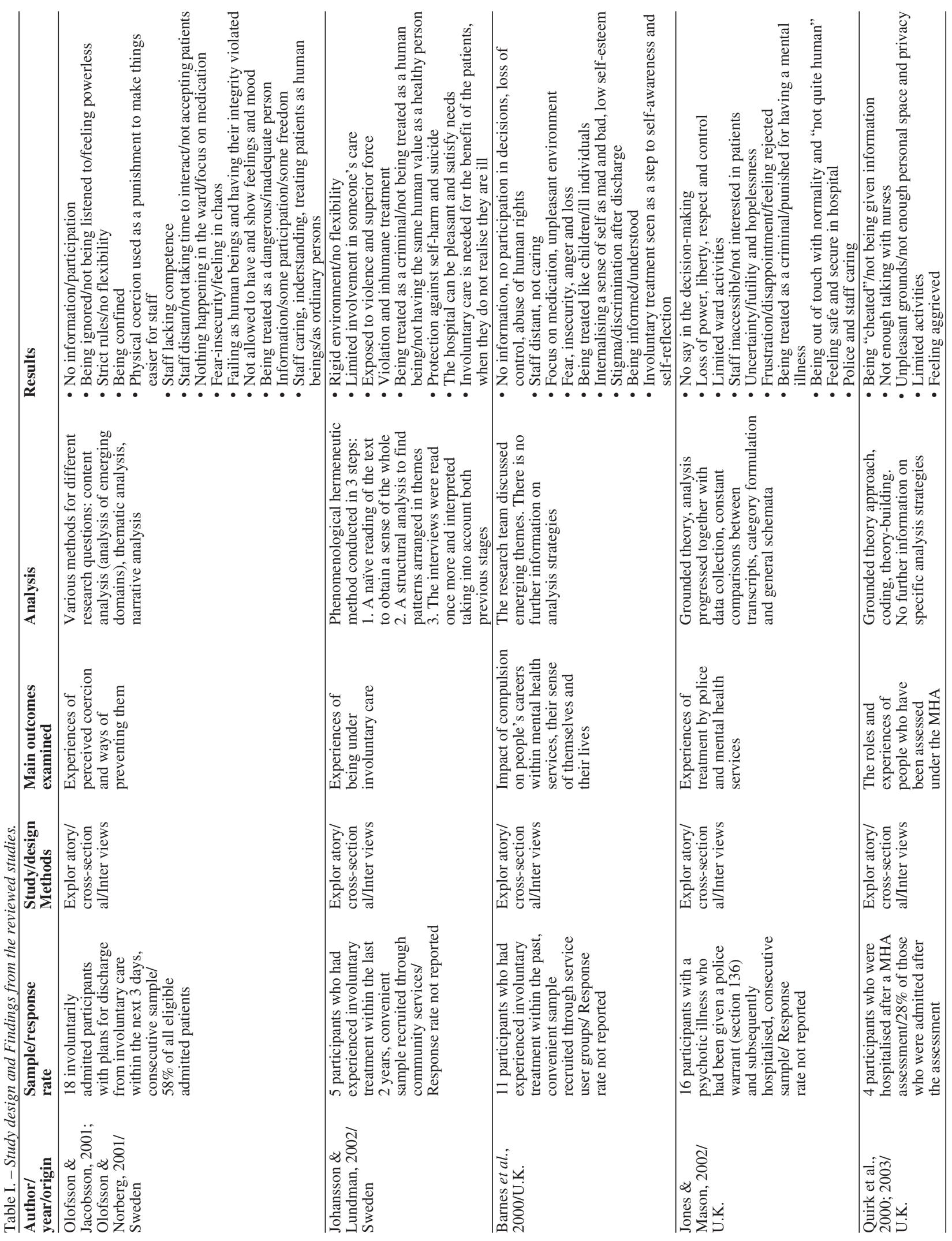

Epidemiologia e Psichiatria Sociale, 16, 2, 2007 
this isn't any direct care. It is on the whole only storing away" (Olofsson \& Jacobsson, 2001). They believe that the staff are not competent enough and they do not take time to listen and talk, they are not caring nor supportive. "I felt so extremely bad and wanted someone to talk to, it was at night I recall. But he said I can't help you, and he just went away, he could at least just sit by my side. Or talk to me about anything then, I don't expect him to do miracles but just being there would have been enough" (Johansson \& Lundman, 2002). In addition, the ward environment is not conductive to their well being, as there are limited activities people can get involved in, other patients can be frightening, the food is not healthy and there are no pleasant grounds or outside spaces. Furthermore, there is no personal space or opportunities for privacy. The only treatment option is medication and this can cause unpleasant side-effects.

\section{c) Emotional impact of involuntary treatment-feeling devalued:}

Patients report that involuntary admission usually causes major disruption to their lives. This often involves being subjected to coercion, physical or psychological, and having to conform with external rules imposed by professionals.

Patients often feel that these professionals are not interested in them, do not accept them as they are and are even irritated by their behaviour. This situation evokes quite strong emotional responses. Patients feel rejected and aggrieved. They are disappointed in the staff and mental health system in general and feel pessimistic about their future. This leads to feelings of hopelessness, frustration, low self-esteem and loss of control over their lives.

These powerful emotions make patients feel insecure about themselves and question their value. They often express that they do not have the same status as a healthy person, as they are being treated like criminals or mentally ill patients. They feel they are punished for having a mental illness and for taking police's and staff's time. Their personal integrity is violated, as they believe they are not treated like human beings. They feel out of touch with normality and dehumanised. "...everybody ought to be valued equally even if you are ill. But sometimes you are made to feel that you are not worth anything, have no human value. (Johansson \& Lundman, 2002). "You become a nobody, they can do whatever they want with you, although maybe you are a very valuable person in a crisis". (Olofsson \& Jacobsson, 2001). Such feelings are sometimes more permanent, leaving people to feel devalued and stigmatised even after discharge.

\section{Positive aspects}

a) Respect-autonomy:

Although patients report violations of their autonomy, some of them also experience that they participate in their treatment planning, they collaborate with health professionals, they are informed about their rights and treatment and they are up to an extent responsible for their care. Although they are kept in a hospital against their will they do not always feel coerced, they are sometimes given more freedom and flexibility, which they appreciate. "...he [the physician] was flexible. So that was very good. I was pleasantly surprised when he released me so soon..." (Johansson \& Lundman, 2002).

\section{b) Being cared for/ treatment benefits:}

In some cases people feel that police and staff are looking after them, taking time to interact with them and are interested in their progress. They believe that some professionals are personally interested in them, take time to talk to them and provide valuable care and support. Such close relationships with staff alleviate patients' feelings of fear and uncertainty. "There was a very nice person taking my hand, showing she really bothered about me, making me feel well. This gave me my security back and I began to talk with the staff. I felt they were like angels, very nice persons and I thanked them for understanding me and bothering about me". (Olofsson \& Jacobsson, 2001). Relationships with other patients and relatives are also important and help patients overcome their problems.

The hospital is often considered to be a place of safety. Some patients retrospectively report that involuntary admission can be justified when they do not realise they are ill and it can offer protection against self- harm, suicide and other risks. In some cases coercion is viewed as necessary and unavoidable. It can also lead to self-awareness, positive treatment outcomes and benefit the patients in the long term. "I thought well at least I 'll get a cigarette and a cup of tea... I mean I could have been left out on the streets and beaten up... you 've got to be thankful at the end of the day because look at me now I 've got a new pair of trousers, new t-shirt... [my care manager] has brought me here and things have brightened up". (Quirk et al., 2000).

\section{Being a human being like other people:}

When patients feel that they are treated as ordinary individuals by staff their self- confidence is enhanced and they feel normal. They feel they are human beings like 
other people and they appreciate being allowed to behave as such, showing feelings and expressing the wish to be looked after. Characteristics that further strengthen their sense of being normal, such as having a house, a job, friends, relatives and pets are also important while under involuntary care.

\section{Review questions 2 and 3}

Although patients' experiences are described in the reviewed studies, it is not clear how positive and negative aspects of involuntary hospitalisation are linked to each other. Although there is a presentation of positive and negative themes regarding involuntary treatment, there is no information on whether these themes were mentioned by different patient groups who are overall negative or positive towards compulsory care, or by the same individuals who can perceive both gains and drawbacks.

Similarly, there is no attempt to identify differences between patient groups who hold different views regarding coercive treatment. Although some of the characteristics of participants are reported in the reviewed studies no possible links between these characteristics and people's views are suggested. Hence, the third review question and part of the second one remain unanswered.

\section{DISCUSSION}

The thematic analysis presented above offers some insight into patients' experiences and perceived outcomes of involuntary care beyond those reported in numerical quantitative studies (Katsakou \& Priebe, 2006). It also illustrates positive and negative aspects of such care, thus answering the first and part of the second of the review questions. It appears that being under involuntary care is a complex process, consisting of different stages, which can elicit various concerns and emotions to people experiencing it. The main areas that involuntary patients are concerned about are violations of their autonomy, the rather low quality of treatment they believe they receive from services and professionals, and the impact of both these situations on their sense of self-value. That is, when people feel that they have no say in the treatment they receive and are treated disrespectfully by staff, their selfconfidence is automatically affected and they feel they are inferior human beings, having less value than other people. Nevertheless, when they feel respected, cared for and given some room for autonomous decisions and participation in their care planning, they feel more normal and being human beings like other people.
However, although the studies reviewed here offer some insight into patients' experiences of involuntary care, they do not address other significant review questions. In effect, it is not described whether the emerging themes are mentioned by all participants as negative and positive aspects of their overall experience or whether they reflect different views supported by different patient groups.

Although for the present investigation a thematic analysis of topics identified in the reviewed studies was performed, this synthesis was performed by one researcher. It has been claimed that the synthesis of qualitative information is more open to biases and subjective interpretation than that of quantitative and numerical information. Nonetheless, the results of the synthesis and the interpretation of findings were discussed in detail with the second reviewer.

In addition, the studies reviewed here were only 5 and all of them had particularly small sample sizes. Hence, the results presented are derived from interviews with only 54 patients in total. Lastly, the methods used are not always clearly described, as reported in the results section, and therefore the quality of the studies is difficult to assess.

However, these findings are in line with findings from other studies examining outcomes of coercive treatment in different settings, such as long-stay psychiatric wards (Pereira et al., 2005), supervised community treatment and guardianship (Canvin et al., 2005), assertive community treatment under assertive outreach teams (Watts \& Priebe, 2002) and acute psychiatric hospital admission among both voluntary and involuntary patients (Bennett et al., 1993). Overall, people suffering from mental health problems are particularly sensitive against any intrusions into their autonomy and privacy. Coercive interventions are often viewed as an attack on their overall ability to self-regulate. On the other hand, when people feel that they are given the chance to participate in decisions regarding their care and that health professionals are genuinely interested in their well being, they find it easier to justify and accept compulsory treatment.

These findings have significant implications for involuntary inpatient care. It is important to understand how vulnerable and insecure people exposed to coercive interventions might be. Violations of autonomy are probably unavoidable when people are treated against their will. However, when patients feel that professionals genuinely care about them and offer them some degree of participation in their treatment, such interventions are viewed in a less negative light and do not have a negative impact on people's sense of self-value. Thus, it seems important to

Epidemiologia e Psichiatria Sociale, 16, 2, 2007 
enhance people's perceived participation even when being treated involuntarily. Provision of information on treatment issues, discussion of possible alternatives and more personal interaction between patients and professionals may prove useful tools towards this goal.

It is important for health services to ensure that the necessity to compulsorily admit and treat people for a short time does not interfere with the long term goal of promoting autonomy and independence, which could be the case if people leave the hospital feeling inadequate and inferior.

Furthermore, possible differences between distinct patient groups should be explored in future research, as they could have different implications for treatment. If people hold both negative and positive views, the goal would be to enhance positive experiences by focusing on respect and autonomy issues. On the other hand, if different patient groups report different opinions, it would be crucial to identify those with poorer outcomes in order to address issues that might be specific to their personal characteristics or to their treatment and design relevant interventions.

Acknowledgements. This review was supported by a grant from the Research Policy Programme of the Department of Health (UK).

\section{REFERENCES}

Barbour R. (2001). Checklists for improving rigour in qualitative research: a case of the tail wagging the dog? British Medical Journal 322, 1115-1117.

Barnes M., Davis A., Tew J., Gell C. Glynn T., Lewis L., Pryce J. \& Saddal S. (2000). Users experiences of compulsion under the 1983 Mental Health Act. University of Birmingham (unpublished report).

Bennett N.S., Lidz C.W., Monahan J., Mulvey E., Hoge S.K., Roth L.H. \& Gardner W. (1993). Inclusion, motivation and good faith: the morality of coercion in mental hospital admission. Behavioural Sciences and the Law 11, 295-306.

Bromley H., Dockery G., Fenton C., Nhelma B., Smith H., Tolhurst R. \& Theobald S. (2002). Criteria for evaluating qualitative studies. Retrieved November 16, 2006, from http:/www.liv.ac.uk/lstm/ download/guidelines.pdf

Canvin K., Bartlett A. \& Pinfold V. (2005). Acceptability of compulsory powers in the community: the ethical considerations of mental health service users on supervised discharge and guardianship. Journal of Medical Ethics 31, 457-462.

Clark J.P. (2003). How to peer review a qualitative manuscript. In Peer Review in Health Sciences. Second edition. (ed. G. F. Jefferson and T. London), pp. 219-235. BMJ Books: London.

Clarke M. (2000). The Quorom statement. Lancet 355 (9205), 756-757.

Dixon- Woods M. \& Fitzpatrick R. (2001). Qualitative research in systematic reviews. British Medical Journal 323, 765-766.

Dixon- Woods M., Shaw R.L., Agarwal S. \& Smith J.A. (2004). The problem of appraising qualitative research. Quality and Safety in Health Care 13, 223-225.

Barnes M., Davis A., Tew J., Gell C. Glynn T., Lewis L., Pryce J. \& Saddal S. (2000). Users' Experiences of Compulsion Under the 1983 Mental Health Act. University of Birmingham (unpublished report).

Johansson I.M. \& Lundman B. (2002). Patients' experiences of involuntary psychiatric care: good opportunities and great losses. Journal of Psychiatric and Mental Health Nursing 9, 639-647.

Jones, S.L. \& Mason, T. (2002). Quality of treatment following police detention of mentally disordered offenders. Journal of Psychiatric and Mental Health Nursing 9, 73-80.

Katsakou C. \& Priebe S. (2006). Outcomes of involuntary hospital admission- a review. Acta Psychiatrica Scandinavica 114 (4), 232241.

Law M., Stewart D., Letts L., Pollock N., Bosch J. \& Westmorland M. (1998). Guidelines for Critical Review Form- Qualitative Studies. Retrieved November 16, 2006, from http://www.usc.edu/hsc/ebnet/ res/Guidelines.pdf

Milton Keynes Primary Care Trust. (2002). Critical Appraisal Skills Programme (CASP). 10 Questions to help you make sense of qualitative research. Retrieved November 16, 2006, from http://www. phru.nhs.uk/casp/casp.htm

Olofsson B. \& Jacobsson L. (2001). A plea for respect: involuntary hospitalized psychiatric patients' narratives about being subjected to coercion. Journal of Psychiatric and mental Health Nursing 8, 357366.

Olofsson B. \& Norberg A. (2001). Experiences of coercion in psychiatric care as narrated by patients, nurses and physicians. Journal of Advanced Nursing 33(1), 89-97.

Pereira M.A.O., Furegato A.R.F. \& Pereira A. (2005). The lived experience of long term psychiatric hospitalization of four women in Brazil. Perspectives in Psychiatric Care 41 (3), 124-132.

Quirk A., Lelliott P., Audini B. \& Buston K. (2000). Module C Performing the Act: A Qualitative Study of the Process of Mental Health Act Assessments. Final Report to the Department of Health, London. (unpublished report)

Quirk A., Lelliott P., Audini B. \& Buston K. (2003). Non-clinical and extra-legal influences on decisions about compulsory admission to psychiatric hospital. Journal of Mental Health 12 (2), 119-130.

Seale C. (2002). Quality issues in qualitative inquiry. Qualitative Social Work 1, 97-110.

Watts J. \& Priebe S. (2002). A phenomenological account of users' experiences of assertive community treatment. Bioethics 16 (5), 439-454. 